\title{
Ocular Syphilis — Eight Jurisdictions, United States, 2014-2015
}

\author{
Sara E. Oliver, $\mathrm{MD}^{1,2}$; Mark Aubin ${ }^{3}$; Leah Atwell, $\mathrm{MPH}^{4}$; James Matthias, $\mathrm{MPH}^{4,5}$; Anna Cope, PhD 5,6 ; Victoria Mobley, $\mathrm{MD}^{6}$; \\ Alexandra Goode, MSc7 ; Sydney Minnerly, MA ${ }^{8}$; Juliet Stoltey, MD 9 ; Heidi M. Bauer, MD 9 ; Robin R. Hennessy, MPH ${ }^{5,10}$; \\ Dawne DiOrio, MPA5,11; Robyn Neblett Fanfair, MD ${ }^{12}$; Thomas A. Peterman, MD ${ }^{5}$; Lauri Markowitz, MD ${ }^{2}$
}

Ocular syphilis, a manifestation of Treponema pallidum infection, can cause a variety of ocular signs and symptoms, including eye redness, blurry vision, and vision loss. Although syphilis is nationally notifiable, ocular manifestations are not reportable to CDC. Syphilis rates have increased in the United States since 2000. After ocular syphilis clusters were reported in early 2015, CDC issued a clinical advisory (1) in April 2015 and published a description of the cases in October 2015 (2). Because of concerns about an increase in ocular syphilis, eight jurisdictions (California, excluding Los Angeles and San Francisco, Florida, Indiana, Maryland, New York City, North Carolina, Texas, and Washington) reviewed syphilis surveillance and case investigation data from 2014, 2015, or both to ascertain syphilis cases with ocular manifestations. A total of 388 suspected ocular syphilis cases were identified, 157 in 2014 and 231 in 2015. Overall, among total syphilis surveillance cases in the jurisdictions evaluated, $0.53 \%$ in 2014 and $0.65 \%$ in 2015 indicated ocular symptoms. Five jurisdictions described an increase in suspected ocular syphilis cases in 2014 and 2015 . The predominance of cases in men (93\%), proportion of those who are men who have sex with men (MSM), and percentage who are HIV-positive (51\%) are consistent with the epidemiology of syphilis in the United States. It is important for clinicians to be aware of potential visual complications related to syphilis infections. Prompt identification of potential ocular syphilis, ophthalmologic evaluation, and appropriate treatment are critical to prevent or manage visual symptoms and sequelae of ocular syphilis.
In early 2015, clusters of ocular syphilis cases were reported in Washington and California. CDC issued a clinical advisory, notifying clinical providers and health departments of a potential increase in suspected ocular syphilis cases. After this advisory, eight jurisdictions performed a review of syphilis surveillance and case investigation data to identify syphilis cases with ocular manifestations. Seven jurisdictions reviewed data from January 1, 2014 to December 31, 2015; Indiana reviewed data from 2015 only. A patient whose illness met the surveillance case definition of syphilis (3) was considered to have a suspected case of ocular syphilis if the patient had concurrent ocular signs or symptoms noted in the surveillance database from a local syphilis case investigation or reported by a local health care provider. A standard form was used to abstract de-identified information on each case, including demographic information, syphilis stage and treatment, and any information on extent of ocular involvement. Each jurisdiction also provided a total number of syphilis surveillance cases, including numbers from all stages of syphilis, as defined by the surveillance case definitions (3).

A total of 388 suspected ocular syphilis cases were identified, 157 cases in 2014 and 231 cases in 2015 (Table 1). Overall, $0.60 \%$ of total syphilis cases were identified as suspected ocular syphilis cases, $0.53 \%$ in 2014 and $0.65 \%$ in 2015 . The percentage of total syphilis cases with ocular manifestation varied by jurisdiction, ranging from $0.17 \%$ to $3.9 \%$. Five jurisdictions described an increase in suspected ocular syphilis cases in 2014 and 2015.

TABLE 1. Suspected ocular syphilis and total syphilis cases — eight jurisdictions, United States, 2014-2015

\begin{tabular}{|c|c|c|c|c|c|c|}
\hline \multirow[b]{2}{*}{ Jurisdiction } & \multicolumn{2}{|c|}{ Suspected ocular syphilis } & \multicolumn{2}{|c|}{ Total surveillance syphilis cases } & \multicolumn{2}{|c|}{$\begin{array}{c}\text { \% surveillance syphilis cases with } \\
\text { suspected ocular syphilis }\end{array}$} \\
\hline & 2014 & 2015 & 2014 & 2015 & 2014 & 2015 \\
\hline California* & 48 & 60 & 6,238 & 7,824 & 0.77 & 0.77 \\
\hline Florida & 10 & 32 & 6,030 & 7,154 & 0.17 & 0.45 \\
\hline Indiana ${ }^{\dagger}$ & - & 8 & - & 714 & - & 1.10 \\
\hline Maryland & 10 & 17 & 1,524 & 1,779 & 0.66 & 0.96 \\
\hline New York City & 14 & 12 & 5,798 & 6,116 & 0.24 & 0.20 \\
\hline North Carolina & 21 & 42 & 1,799 & 2,435 & 1.20 & 1.70 \\
\hline Texas & 27 & 16 & 7,337 & 8,400 & 0.37 & 0.19 \\
\hline Washington & 27 & 44 & 857 & 1,125 & 3.20 & 3.90 \\
\hline Total & 157 & 231 & 29,583 & 35,547 & 0.53 & 0.65 \\
\hline
\end{tabular}

* California does not include syphilis reports from San Francisco or Los Angeles.

${ }^{+}$Indiana reviewed data from 2015 only. 
Most patients with suspected ocular syphilis were male (93\%), and $249(69 \%)$ of those with information on sex partners were MSM (Table 2). The mean age of patients was 44 years (range $=17-79$ years). Approximately one half of the cases met surveillance criteria for early syphilis (primary, secondary, and early latent syphilis) (Table 3); stage of syphilis was not associated with any specific symptom, diagnosis, or extent of eye involvement. Overall, patients with suspected ocular syphilis had high rapid plasma reagin (RPR) titers, with a median titer of 128 (range $=1-16,384)$. Approximately $22 \%$ of patients reported additional symptoms of neurosyphilis, including headache, neck pain, altered mental status, or changes in hearing.

Specific symptoms were reported by $326(84 \%)$ persons suspected of having ocular syphilis; $54 \%$ of patients reported blurry vision, and $28 \%$ of patients reported at least some vision loss. Specific ocular diagnoses were available for 158 (41\%) patients, and uveitis $(\mathrm{n}=72)$ was the most common diagnosis. More serious diagnoses were also recorded, including retinitis $(n=20)$, optic neuritis $(n=18)$, and retinal detachment $(n=6)$. Of $136(35 \%)$ patients with available information on which eye was affected, one eye was involved in 64 (47\%) patients, and both eyes were affected in $72(53 \%)$ patients.

Among 174 patients with cerebrospinal fluid (CSF) test results, $122(70 \%)$ had a reactive CSF Venereal Disease Research Laboratory (VDRL) test. Patients with a reactive CSF VDRL test were not more likely than patients with a nonreactive CSF VDRL to report additional neurologic symptoms, have vision loss or bilateral eye involvement, or be diagnosed with severe disease, including retinitis, optic neuritis, or retinal detachment.

Recommended treatment for neurosyphilis and ocular syphilis is 18-24 million units intravenous (IV) aqueous penicillin G, administered daily as a continuous infusion, or divided into every 4-hour dosing, for 10-14 days (4). Approximately $60 \%$ of patients with suspected ocular syphilis received IV penicillin, and the other $38 \%$ received varied treatments, most commonly benzathine penicillin given as an intramuscular injection (Table 3).

One half of patients with suspected ocular syphilis were HIV-positive ( $\mathrm{n}=198)$. Of those persons, $62(32 \%)$ were first diagnosed with HIV at the time of their ocular syphilis diagnosis. Compared with HIV-negative patients, HIVpositive patients had a higher median RPR titer (256 versus $128, \mathrm{p}<0.001)$, more often received a lumbar puncture $(57.1 \%$ versus $40.8 \%, \mathrm{p}=0.005)$, and were more often treated with IV penicillin $(66.7 \%$ versus $44.2 \%, \mathrm{p}<0.001)$. Patients with HIV infection did not differ significantly from HIVnegative patients in proportion having a reactive CSF VDRL, both eyes involved, additional symptoms of neurosyphilis,
TABLE 2. Demographic characteristics of patients with suspected ocular syphilis - eight jurisdictions, United States, 2014-2015

\begin{tabular}{lrr}
\hline Characteristic & No. & \multicolumn{1}{c}{$(\%)$} \\
\hline Total & 388 & $(100.0)$ \\
Male & 362 & $(93.3)$ \\
Known MSM (among 362 males) & 249 & $(68.8)$ \\
Race & & \\
White & 217 & $(55.9)$ \\
Black & 81 & $(20.9)$ \\
Hispanic & 48 & $(12.4)$ \\
Asian & 13 & $(3.4)$ \\
Native Hawaiian/Pacific Islander & 1 & $(0.3)$ \\
Other/Unknown & 28 & $(7.2)$ \\
HIV-positive & 198 & $(51.0)$ \\
\hline
\end{tabular}

Abbreviations: $\mathrm{HIV}=$ human immunodeficiency virus; $\mathrm{MSM}=$ men who have sex with men.

or an ophthalmologic exam. CD4 count was available for 126 patients; $84(67 \%)$ had a CD4 count $<500$ cells $/ \mu \mathrm{L}$. Compared with patients with higher CD4 counts, those with a CD4 count $<500$ cells $/ \mu \mathrm{L}$ more often received a lumbar puncture $(65.5 \%$ versus $45.2 \%, \mathrm{p}=0.03)$, had a reactive CSF VDRL $(84.0 \%$ versus $50.0 \%, \mathrm{p}=0.006)$, and had both eyes involved $(35.7 \%$ versus $7.1 \%, \mathrm{p}=0.003)$, but did not differ in proportion with blurry vision or vision loss.

\section{Discussion}

Ocular syphilis is a serious manifestation of syphilis. This report is the first evaluating suspected ocular syphilis across multiple jurisdictions in the United States. Although there is no national reporting of ocular manifestations, eight jurisdictions reviewed their syphilis surveillance data to identify cases with ocular manifestations in 2014 and 2015. Ocular manifestations were present in $0.60 \%$ of all reported syphilis cases, ranging by jurisdiction from $0.17 \%$ to $3.9 \%$. In most jurisdictions, the percentage was similar to data from a study in England that estimated ocular syphilis affected approximately $0.6 \%$ of early syphilis cases from 2009 to 2010 (5). Five of the seven jurisdictions that reviewed cases in both years described an increase in suspected ocular syphilis cases in 2014 and 2015. In addition, after the clinical advisory, CDC was notified of suspected ocular syphilis cases from 20 states (2). The number of cases with ocular syphilis detected in 2014 and 2015 in the United States could be attributable to increased recognition of ocular manifestations in the setting of increased syphilis rates, or an actual increase in the proportion of syphilis cases with ocular disease. The predominance of cases in men and MSM, as well as the proportion also diagnosed with HIV, is consistent with the epidemiology of syphilis in the United States $(\sigma)$

Although two of the cases from Washington reported in the October 2015 MMWR (2) were in sex partners, no suspected 
TABLE 3. Clinical characteristics, laboratory results and diagnoses for syphilis and suspected ocular syphilis - eight jurisdictions, United States, 2014-2015

\begin{tabular}{lrr}
\hline Characteristic & No. & $(\%)$ \\
\hline Total & 388 & $(100.0)$ \\
Stage of syphilis & & \\
Primary & 8 & $(2.1)$ \\
Secondary & 101 & $(26.0)$ \\
Early latent & 79 & $(20.4)$ \\
Late or latent of unknown duration & 193 & $(49.7)$ \\
Unknown & 7 & $(1.8)$ \\
Additional symptoms of neurosyphilis & 87 & $(22.4)$ \\
Reported ocular symptoms (among 326 with symptoms) & \\
Blurry vision & 210 & $(64.4)$ \\
Vision loss & 107 & $(32.8)$ \\
Eye pain or red eye & 46 & $(14.1)$ \\
Eye exam & 158 & $(40.7)$ \\
Diagnosis (among 158 with documented eye exam)* & \\
Uveitis & 72 & $(45.6)$ \\
Retinitis & 20 & $(12.7)$ \\
Optic neuritis & 18 & $(11.4)$ \\
Retinal detachment & 6 & $(3.8)$ \\
CSF analysis performed & 188 & $(48.5)$ \\
CSF VDRL (among 174 with a documented result) & & \\
Reactive & 122 & $(70.1)$ \\
Nonreactive & 52 & $(29.9)$ \\
Treatment & & \\
Aqueous penicillin G IV & 230 & $(59.3)$ \\
Other treatment & 146 & $(37.6)$ \\
No/Unknown treatment & 12 & $(3.1)$ \\
\hline Abbreviations: CSF & & \\
\hline
\end{tabular}

Abbreviations: $\mathrm{CSF}=$ cerebrospinal fluid; IV = intravenous; VDRL = Venereal Disease Research Laboratory test.

* Can be included in multiple categories.

ocular syphilis cases in North Carolina, Indiana, New York City, or Florida named a person with ocular syphilis as a sex partner; data from other jurisdictions were unavailable. In addition, in a preliminary study, no specific strain was identified in patients with ocular syphilis, suggesting a single oculotropic strain of T. pallidum is not responsible for the apparent increase in ocular syphilis (7). The absence of both a specific strain and epidemiologic links supports a hypothesis that manifestations of ocular syphilis occur in a subset of patients with syphilis infection, possibly influenced by undetermined risk factors.

The findings in this report are subject to at least five limitations. First, each jurisdiction had slightly different methods for identifying patients with possible ocular manifestations of syphilis; capacity and infrastructure to investigate syphilis vary by state as well. Second, patients with ocular syphilis would have been missed if ocular symptoms were not documented in the case investigation, or if a case investigation was not completed per local investigation protocols. Therefore, the numbers described here could either underrepresent or overrepresent the burden of ocular syphilis. Third, a majority of jurisdictions did not request medical charts to confirm that

\begin{abstract}
Summary
What is already known about this topic?

Ocular syphilis, an infrequent manifestation of syphilis infection, can cause a variety of eye symptoms, including vision loss. Clusters of ocular syphilis were reported from late 2014 to 2015. In the United States, syphilis rates have increased since 2000, but little is known about ocular syphilis cases.

What is added by this report?

Eight jurisdictions that reviewed syphilis surveillance and case investigation data from 2014,2015 , or both found that in $0.6 \%$ of syphilis cases, the patient had symptoms consistent with ocular syphilis. Most suspected cases were in males, and half were in HIV-positive persons. Severe outcomes, including blindness, occurred in both HIV-positive and HIV-negative patients.

What are the implications for public health practice?

All patients diagnosed with syphilis that exhibit ocular manifestations should immediately be treated for neurosyphilis and be referred for formal ophthalmologic examination. Education of both patients and providers is critical to identify ocular manifestations of syphilis and manage disease sequelae.
\end{abstract}

ocular symptoms were syphilis-related; therefore, some symptoms could have causes unrelated to syphilis infection. Fourth, many cases had incomplete information provided as specific ocular diagnoses are not routinely collected as part of syphilis case investigations. Finally, trends over time cannot be assessed because information about suspected ocular syphilis was not available for years before 2014 .

Public health interventions aimed at both providers and persons at risk are necessary to prevent ocular syphilis, and to ensure prompt diagnosis and treatment. All patients diagnosed with syphilis that exhibit ocular manifestations, such as eye pain, blurry vision, or vision loss, should immediately be treated for neurosyphilis, and be referred for expert ophthalmologic examination. Severe outcomes, including blindness, occur in both HIV-positive and HIV-negative patients. Further investigation is currently underway to identify additional risk factors specific to ocular syphilis. Because the prevalence of syphilis is increasing in the United States, education of both patients and providers is critical to identify ocular manifestations of syphilis as early as possible and manage disease sequelae.

\section{Acknowledgments}

Local and state health departments; disease investigation specialists; Sandra Matus, Center for STI Prevention, Maryland Department of Health and Mental Hygiene; Denise Gilson and Michael Samuel, Sexually Transmitted Disease Control Branch, California Department of Public Health; Elissa Meites and Julia Gargano, Division of Viral Diseases, National Center for Immunization and Respiratory Diseases, CDC. 


\begin{abstract}
${ }^{1}$ Epidemic Intelligence Service, CDC; ${ }^{2}$ Division of Viral Diseases, National Center for Immunization and Respiratory Diseases, CDC; ${ }^{3}$ STD Services, Washington State Department of Health; ${ }^{4}$ Bureau of Communicable Diseases, Florida Department of Health; ${ }^{5}$ Division of STD Prevention, National Center for HIV/AIDS, Viral Hepatitis, STD and TB Prevention, CDC; ${ }^{6}$ Division of Public Health, North Carolina Department of Health and Human Services; ${ }^{7}$ Center for STI Prevention, Maryland Department of Health and Mental Hygiene; ${ }^{8} \mathrm{HIV} / \mathrm{STD} / \mathrm{TB} /$ Viral Hepatitis Prevention Branch, Department of State Health Services; ${ }^{9}$ Sexually Transmitted Disease Control Branch, California Department of Public Health; ${ }^{10}$ Bureau of STD Control, New York City Department of Health and Mental Hygiene; ${ }^{11} \mathrm{HIV} / S T D$ Division, Indiana State Department of Health; ${ }^{12}$ Division of HIV/AIDS Prevention, National Center for HIV/AIDS, Viral Hepatitis, STD and TB Prevention, CDC.
\end{abstract}

\section{References}

1. CDC. Clinical advisory: ocular syphilis in the United States 2015. Atlanta, GA: US Health and Human Services, CDC; 2016. http://www.cdc.gov/ std/syphilis/clinicaladvisoryos2015.htm

2. Woolston S, Cohen SE, Fanfair RN, Lewis SC, Marra CM, Golden MR. A cluster of ocular syphilis cases-Seattle, Washington, and San Francisco, California, 2014-2015. MMWR Morb Mortal Wkly Rep 2015;64:1150-1. http://dx.doi.org/10.15585/mmwr.mm6440a6

3. CDC. STD surveillance case definitions. Atlanta, GA: US Health and Human Services, CDC; 2016. http://www.cdc.gov/std/stats10/app-casedef.htm

4. Workowski KA, Bolan GA; CDC. Sexually transmitted diseases treatment guidelines, 2015. MMWR Recomm Rep 2015;64(No. RR-03).

5. Mathew RG, Goh BT, Westcott MC. British Ocular Syphilis Study (BOSS): 2-year national surveillance study of intraocular inflammation secondary to ocular syphilis. Invest Ophthalmol Vis Sci 2014;55:53945400. http://dx.doi.org/10.1167/iovs.14-14559

6. CDC. Sexually transmitted disease surveillance 2014. Atlanta, GA: US Department of Health and Human Services, CDC; 2015. http://www. cdc.gov/std/stats14/default.htm

7. Oliver S, Sahi SK, Tantalo LC, et al. Molecular typing of Treponema pallidum in ocular syphilis. Sex Transm Dis 2016;43:524-7. http://dx.doi. org/10.1097/OLQ.0000000000000478 